Www.jmscr.igmpublication.org

Index Copernicus Value: 79.54

ISSN (e)-2347-176x ISSN (p) 2455-0450

crossref DOI: https://dx.doi.org/10.18535/jmscr/v7i4.27

Journal Of Medical Science And Clinical Research

IGM Publication

An official Publication of IGM Publication

\title{
Comparative Evaluation of Stannous Fluoride and Arginine Bicarbonate Desensitizer in the Reduction of Dentinal Hypersensitivity after Ultrasonic Scaling - A Randomized Controlled Trial
}

\author{
Authors \\ Yogalakshmi Suubha $^{1}$, Vijayalakshmi ${ }^{2}$, Ambalavanan $^{3}$, L. Karthik $^{* 4}$ \\ ${ }^{1,2,3}$ Department of Periodontology, Meenakshi Ammal Dental College \& Hospital,Chennai, Tamil Nadu, \\ India - 600095 \\ ${ }^{4}$ Meenakshi Academy of Higher Education and Research, K.K. Nagar West, Chennai, Tamil Nadu, \\ India - 600078 \\ *Corresponding Author \\ L. Karthik \\ Email: karthikmaher85@gmail.com, Contact number: +91-9791722707
}

\begin{abstract}
Aim: The aim of this study was to evaluate and compare the efficacy of two desensitizing agents containing stannous fluoride and arginine bicarbonate in the reduction of dentinal hypersensitivity after ultrasonic scaling.

Materials and Methods: The subjects for this study were recruited from the outpatient department of Periodontology. About 90 subjects reported with the complaint of hypersensitivity immediately after ultrasonic scaling were chosen for the study. Based on the inclusion and exclusion criteria they were randomized into 3 groups with 30 patients representing each group. The study was approved by the "Institutional Review Board". The patients were explained about the study and written informed consent was obtained from those who agreed to voluntarily participate in this study.

Results: Intergroup comparison among the three groups have revealed that dentin hypersensitivity in Group A (stannous fluoride) was statistically significant when compared to that of Group B (arginine bicarbonate) at $3^{\text {rd }}$ and $7^{\text {th }}$ day. The Visual Analogue Score (VAS) of Group A was found to be significantly lower than the VAS score of Group B.

Conclusion: It was concluded that compared to arginine bicarbonate, stannous fluoride has exhibited better effect in managing dentinal hypersensitivity.

Keywords: Arginine Bicarbonate; Dentinal Hypersensitivity; Desensitizer; Stannous Fluoride; Ultrasonic Scaling.
\end{abstract}

\section{Introduction}

Dentin sensitivity, cervical dentinal sensitivity or dentinal hypersensitivity is one of the most commonly encountered clinical problems. It is clinically described as an exaggerated response to application of a stimulus to exposed dentin, regardless of its location ${ }^{(1,2)}$.The terms dentin sensitivity or dentinal hypersensitivity have been used interchangeably to describe the same clinical condition. True hypersensitivity can develop due to pulpal inflammation and can present the clinical features of irreversible pulpitis, i.e., severe and 
persistent pain, as compared with typical short sharp pain of dentinal hypersensitivity ${ }^{(3)}$. Majority of literature reviews dealing with this clinical condition have suggested the use of term dentin sensitivity and consider that the sharp pain is actually the normal pulpal response to the exposed dentin $^{(4)}$. Dentin may become exposed via several means such as enamel or cementum which normally covers the dentin surface that can be removed or denuded as a result of attrition, abrasion or erosion. Alternatively, in some individuals the cementum and enamel which normally cover the dentin do not meet and result in dentin exposure as a result of a developmental anomaly.

In general, it appears that dentinal hypersensitivity is rarely a result of just one of the above factors, but rather a combination of more than one factor. Regardless of the aetiology of dentin exposure, one feature appears to be in common and that is open dentinal tubules which provide a direct link between the external environment and the internal pulp of the tooth. If the tubules are not exposed it seems unlikely that hypersensitivity will occur. Even after longer periods of exposure to the oral environment, dentinal sensitivity may still be a significant problem despite the exposed tubules becoming occluded by the smear layer or pellicle. Thus, once sensitivity has become established the pulp may become irreversibly sensitive. The problem of dentin hypersensitivity that affects up to57\% of patients represents actually a main concern in daily dental care because of lack of effective treatments ${ }^{(5)}$. The fore most symptom reported by patients particularly after scaling and root planning is pain provoked by external, thermal, mechanical, osmotic stimuli as well as chemical and not related to dental pathology due to pulpal pathology, fractured teeth, carious lesions, leaking restorations or palatogingival grooves $^{(6)}$. Dentin can become exposed to the buccal cavity due to two main reasons: 1) When the enamel wears out by abrasion, attrition, erosion or abfraction ${ }^{(7)}$ and 2) Through gingival recession due to age, periodontal pathologies, incorrect brushing techniques or use of inadequate toothbrushes, often hard bristled ${ }^{(8)}$.

The most common way is by prescribing toothpastes containing potassium salts known for its "numbing" action through depolarization on the afferent nerve at the end of dentinal tubules ${ }^{(9)}$. Most products require continued use over a 4 to 8 weeks period before considerable relief could be achieved. An implicit consequence of the hydrodynamic theory is the polarisation of treatment options into those that target desensitization of the relevant intradental nerves and those whose aim is to inhibit or prevent transmission of the stimulus itself. Stannous fluoride formulations have been shown to provide significant reduction in dentin hypersensitivity after 4 or more weeks of twice daily use. A desensitizing agent containing $0.454 \%$ stannous fluoride was shown to significantly reduce sensitivity, on both tactile and air blast measures, compared to sodium fluoride, after 4 and 8 weeks use. The highly soluble arginine bicarbonate surrounds the particles of the poorly soluble calcium carbonate forming an alkaline paste-like complex. This "super saliva spackle" will immediately plug and seal off a tooth's open dentinal tubules, thereby eliminating or reducing dentinal sensitivity following scaling. This study was designed with an aim to evaluate the efficacy of stannous fluoride vs arginine bicarbonate for the treatment of dentinal hypersensitivity following ultrasonic scaling.

\section{Materials and Methods}

The subjects for this study were recruited from the outpatient department of Periodontology. About 90 subjects were selected with the complaint of hypersensitivity immediately after ultrasonic scaling and randomized into 3 groups based on the inclusion and exclusion criteria. The study was approved by the "Institutional Review Board". The patients were explained about the study and written informed consent was obtained from those who agreed to voluntarily participate in this study. 


\section{Selection Criteria}

The study subjects for the present study were chosen based on the following criteria.1) Patients who had undergone ultrasonic scaling and presenting with a Visual Analogue Score(VAS) score $>5$ immediately after ultrasonic scaling and 2)Patients who were found with the presence of upper and lower anteriors. Patients satisfying the above criteria were included for the study. Similarly, patients reported with the following conditions such as 1) Cervical abrasion, 2) Cervical erosion, 3) Dental Caries, 4) Restorations, 5) Crown and 6) Dentures were excluded from the study.

\section{Sample Selection}

A total of 90 patients fulfilling the inclusion criteria were chosen for the study. With 30 patients in each group they were randomly divided into 3 groups as Group A, Group B and Group C using a computer generated program. Informed consent was obtained from all the patients.

\section{Test Groups - Group A and Group B}

A total of 60 patients were randomly categorised into two groups. In both the groups the patients were made to undergo ultrasonic scaling. Following this, while the patients belong to Group A were provided with stannous fluoride, patients belong to Group B were provided with arginine bicarbonate tooth paste for the relief of dentinal hypersensitivity caused due to ultrasonic scaling.

\section{Control Group - Group C}

For the 30 patients in the control group (i.e. Group C) after undergoing ultrasonic scaling, they were supplied with immediate in-office application of 'non-fluoridated' toothpaste containing no desensitizing agent.

\section{Procedure - VAS Scale for Cold Air Stimulus}

After completion of ultrasonic scaling, clinical examination was carried out in a dental chair under standard conditions of light. Dentinal hypersensitivity was scored using VAS scale on cold air stimulus. A method used for measuring the pain, VAS scale is a line of $10 \mathrm{~cm}$ in length where each line represents the limit of pain which the patient might experience from any external stimulus. Subjects were asked to place a mark on the $10 \mathrm{~cm}$ line indicating the intensity of their current level of dentin hypersensitivity. The cold air stimulus is approximately a blast of air from the air syringe of the dental unit for few seconds. The cold air was directed at the exposed surface of the selected tooth that was isolated from the adjacent teeth, using cotton rolls. A constant distance $(3 \mathrm{~mm})$ was kept between the tooth surface and the tip of the air syringe for the assessment of hypersensitivity. The subjects will be asked to mark the response to the stimulus on the VAS scale.

\section{Method of Application}

The upper and lower anteriors were isolated with cotton rolls. The desensitizing agent was applied in the facial and palatal surfaces using a cotton applicator tip and allowed to remain for 3 minutes. Later the patient was instructed to rinse the mouth. Subjects were advised to use the toothpaste with a soft bristle toothbrush twice a day. Subjects were also directed to refrain from any other dentifrice or mouth rinse during the trial, but were allowed to continue their normal oral hygiene practice.

Subjects were recalled on $3^{\text {rd }}$ and $7^{\text {th }}$ day. Hypersensitivity scores were recorded again on cold air stimulus as mentioned above.

\section{Statistical Analysis}

Statistical analysis was done using Statistical Package for Social Sciences (SPSS) version 16.0 (IBM Corp, Chicago, IL, USA). Mean and standard deviation were estimated for both groups. Mean values were compared between different time points within each group by ANOVA and Repeated Measures Analysis of Variance (rANOVA) test. Whereas, mean values between the two groups was compared using Tukey HSD test. In the present study, $p \leq 0.05$ was considered as the level of significance. 


\section{Results}

The purpose of this study was to compare the efficacy of two desensitizing agents containing stannous fluoride and arginine bicarbonate in the reduction of dentinal hypersensitivity after ultrasonic scaling. The study sample consisted of 90 patients who had undergone ultrasonic scaling and obtained a VAS score $>5$ immediately after undergoing ultrasonic scaling. They were randomly divided into three groups: Group A, B and $\mathrm{C}$ consisted of 30 patients each supplied with stannous chloride, arginine bicarbonate and nonfluoridated toothpaste respectively. The results obtained were tabulated and compared statistically.

In Group A, the mean VAS score for sensitivity at baseline was found to be $4.83 \pm 1.34$ (Table 1). On $3^{\text {rd }}$ day, there was a reduction in dentinal sensitivity (DS) to $1.89 \pm 1.34$ and on $7^{\mathrm{t} h}$ day, there was further reduction in DS to $0.24 \pm 0.32$, both of which were statistically significant compared to baseline ( $p$ < 0.05). For Group B, the mean VAS score for sensitivity was $5.03 \pm 1.22$ at baseline (Table 1). On $3^{\text {rd }}$ day, there was a reduction in DS to $3.12 \pm 1.17$ and on $7^{\text {th }}$ day, there was a further reduction in DS to $1.47 \pm 0.94$, both of which were statistically significant compared to baseline $(\mathrm{p}<0.05)$. Similarly, the mean VAS score for sensitivity was observed to be $4.78 \pm 1.58$ for group $\mathrm{C}$ at baseline. As seen in the other 2 groups, in Group $\mathrm{C}$ also a reduction was observed in DS on $3^{\text {rd }}$ day with the mean value of $3.45 \pm$ 1.27. On $7^{\text {th }}$ day, there was a further reduction in DS to $2.77 \pm 1.22$, both of which were statistically significant compared to baseline $(p<0.05)$.

While the mean difference in VAS score for sensitivity between baseline and $3^{\text {rd }}$ day was found to be $2.94 \pm 0.24$, the mean difference in VAS score for sensitivity between baseline and $7^{\text {th }}$ day was found to be $4.59 \pm 0.21$ (Table 2). Similarly, the mean difference in VAS score for sensitivity between $3^{\text {rd }}$ day and $7^{\text {th }}$ day was observed as $1.66 \pm 0.22$ all of which were statistically significant $(p<0.05$, Table 2$)$. The pair wise comparison of Group B analysis have revealed that the mean difference in VAS score obtained for sensitivity between baseline to $3^{\text {rd }}$ day and baseline to $7^{\text {th }}$ day were found to be $1.90 \pm 0.09$ and $3.56 \pm 0.16$ respectively (Table 3 ). The mean difference in VAS score for sensitivity between $3^{\text {rd }}$ day and $7^{\text {th }}$ day was $1.66 \pm 0.12$ all of which were statistically found to be significant $(\mathrm{p}<0.05$, Table 3).

As performed for Group A and B, analysis of the pair wise comparison of Group $\mathrm{C}$ (control) have revealed that the mean difference in VAS score obtained for sensitivity between baseline and $3^{\text {rd }}$ day as well as between baseline and $7^{\text {th }}$ day were found to be $1.33 \pm 0.12$ and $2 \pm 0.12$ respectively (Table 4). The mean difference in VAS score for sensitivity between $3^{\text {rd }}$ day and $7^{\text {th }}$ day was $0.67 \pm 0.05$ all of which were statistically found to be significant $(\mathrm{p}<0.05$, Table 4$)$.

The mean VAS score recorded at the 3rd day was found to be highest in Group $\mathrm{C}$ possessing a mean value of $3.45 \pm 1.26$ followed by Group B and A exhibiting mean values of $3.12 \pm 1.17$ and $1.89 \pm 1.34$ respectively (Table 5).This difference was found to be statistically significant $(\mathrm{p}<0.05$, Table 5). Likewise, the mean VAS score recorded at the 7th day was found to be highest in group $\mathrm{C}$ $(2.77 \pm 1.22)$ followed by group $\mathrm{B}(1.47 \pm 0.94)$ and A $(0.24 \pm 0.32)$. This difference was also found to be statistically significant $(\mathrm{p}<0.05$, Table 5). From pair wise comparison on $3^{\text {rd }}$ day, it was noted that the mean difference in the VAS scores between Group B and C $(-0.32 \pm 0.33)$ was statistically insignificant $(\mathrm{p}=0.586$, Table 6). However, the mean difference between Group A and $\mathrm{B}(-1.23 \pm 0.33)$ as well as Group A and C ($1.55 \pm 0.33)$ were found to be statistically significant $(\mathrm{p}<0.05)$. Similarly, analysis of pair wise comparison on $7^{\text {th }}$ day have showed that the mean difference in the VAS scores between Group A and B (-1.23 \pm 0.24$)$, Group A and C ($2.54 \pm 0.24)$ along with Group B and C (-1.30 \pm 0.23 ) were found to be statistically significant ( $p$ $<0.05$, Table 6). 
Table 1: Intra group Comparison of Groups A, B and C with Respect to VAS (rANOVA) test

\begin{tabular}{|l|c|c|c|c|}
\hline \multirow{2}{*}{ Groups } & \multicolumn{3}{|c|}{ Mean \pm Standard Deviation } & \multirow{2}{*}{ P value } \\
\cline { 2 - 4 } & Baseline & $\mathbf{3}^{\text {rd }}$ day & $\mathbf{7}^{\text {th }}$ day & $p \leq 0.05$ \\
\hline Group A & $4.83 \pm 1.34$ & $1.89 \pm 1.34$ & $0.24 \pm 0.32$ & $0.000^{*}$ \\
\hline Group B & $5.03 \pm 1.22$ & $3.12 \pm 1.17$ & $1.47 \pm 0.94$ & $0.000^{*}$ \\
\hline Group C & $4.78 \pm 1.58$ & $3.45 \pm 1.27$ & $2.77 \pm 1.22$ & $0.000^{*}$ \\
\hline
\end{tabular}

Table 2: Pair wise Intra group Comparison of Group A with Respect to VAS for Air Blast Test Scores at Baseline, $3^{\text {rd }}$ and $7^{\text {th }}$ day by rANOVA Test

\begin{tabular}{|l|c|c|c|}
\hline \multirow{2}{*}{$\begin{array}{l}\text { Time } \\
\text { Points }\end{array}$} & Intragroup Comparison & Mean \pm Standard Deviation & $\begin{array}{l}\boldsymbol{P} \text { value } \\
p \leq 0.05\end{array}$ \\
\hline \multirow{3}{*}{ Baseline } & $3^{\text {rd }}$ day & $2.94 \pm 0.24$ & $0.0001^{*}$ \\
\cline { 2 - 4 } & $7^{\text {th }}$ day & $4.59 \pm 0.21$ & $0.0001^{*}$ \\
\hline \multirow{3}{*}{$3^{\text {rd }}$ day } & Baseline & $-2.94 \pm 0.24$ & $0.0001^{*}$ \\
\cline { 2 - 4 } & $7^{\text {th }}$ day & $1.66 \pm 0.22$ & $0.0001^{*}$ \\
\cline { 2 - 4 } $7^{\text {th }}$ day & Baseline & $-4.59 \pm 0.21$ & $0.0001^{*}$ \\
\cline { 2 - 4 } & $3^{\text {rd }}$ day & $-1.66 \pm 0.22$ & $0.0001^{*}$ \\
\hline
\end{tabular}

Based on estimated marginal means, $*$ - The mean difference is significant at the 0.05 level.

Table 3: Pair wise Intra group Comparison of Group B with Respect to VAS for Air Blast Test Scores at Baseline, $3^{\text {rd }}$ and $7^{\text {th }}$ day by rANOVA Test

\begin{tabular}{|c|c|c|c|}
\hline $\begin{array}{l}\text { Time } \\
\text { Points }\end{array}$ & Intra group Comparison & Mean \pm Standard Deviation & $\begin{array}{l}P \text { value } \\
p \leq 0.05\end{array}$ \\
\hline \multirow[b]{2}{*}{ Baseline } & $3^{\text {rd }}$ day & $1.90 \pm 0.09$ & $0.0001^{*}$ \\
\hline & $7^{\text {th }}$ day & $3.56 \pm 0.16$ & $0.0001 *$ \\
\hline \multirow[b]{2}{*}{$3^{\text {rd }}$ day } & Baseline & $-1.90 \pm 0.09$ & $0.0001^{*}$ \\
\hline & $7^{\text {th }}$ day & $1.66 \pm 0.12$ & $0.0001 *$ \\
\hline \multirow[b]{2}{*}{$7^{\text {th }}$ day } & Baseline & $-3.56 \pm 0.16$ & $0.0001 *$ \\
\hline & $3^{\text {rd }}$ day & $-1.66 \pm 0.12$ & $0.0001 *$ \\
\hline
\end{tabular}

Based on estimated marginal means, * - The mean difference is significant at the 0.05 level.

Table 4: Pair wise Intra group Comparison of Group C with Respect to VAS for Air Blast Test Scores at Baseline, $3^{\text {rd }}$ and $7^{\text {th }}$ day by rANOVA Test

\begin{tabular}{|l|c|c|c|}
\hline \multirow{2}{*}{$\begin{array}{l}\text { Time } \\
\text { Points }\end{array}$} & Intra group Comparison & Mean \pm Standard Deviation & $\begin{array}{l}\boldsymbol{P} \text { value } \\
p \leq 0.05\end{array}$ \\
\hline \multirow{3}{*}{ Baseline } & $3^{\text {rd }}$ day & $1.33 \pm 0.12$ & $0.0001^{*}$ \\
\cline { 2 - 4 } & $7^{\text {th }}$ day & $2 \pm 0.12$ & $0.0001^{*}$ \\
\cline { 2 - 4 } $3^{\text {rd }}$ day & Baseline & $-1.33 \pm 0.12$ & $0.0001^{*}$ \\
\hline \multirow{3}{*}{$7^{\text {th }}$ day } & $7^{\text {th }}$ day & $0.67 \pm 0.05$ & $0.0001^{*}$ \\
\cline { 2 - 4 } & Baseline & $-2 \pm 0.12$ & $0.0001^{*}$ \\
\hline
\end{tabular}

Based on estimated marginal means, * - The mean difference is significant at the 0.05 level

Table 5: Intergroup Comparison of Groups A, B and C with Respect to VAS (rANOVA) test

\begin{tabular}{|c|c|c|c|c|}
\hline \multirow[t]{2}{*}{ Time Points } & \multicolumn{3}{|c|}{ VAS Score Mean \pm Standard Deviation } & \multirow{2}{*}{$\begin{array}{l}P \text { value } \\
p \leq 0.05\end{array}$} \\
\hline & Group A & Group B & Group C & \\
\hline Baseline & $4.83 \pm 1.34$ & $5.03 \pm 1.22$ & $4.78 \pm 1.58$ & 0.768 \\
\hline $3^{\text {rd }}$ day & $1.89 \pm 1.34$ & $3.12 \pm 1.17$ & $3.45 \pm 1.27$ & $0.0001^{*}$ \\
\hline $7^{\text {th }}$ day & $0.24 \pm 0.32$ & $1.47 \pm 0.94$ & $2.77 \pm 1.22$ & $0.0001 *$ \\
\hline
\end{tabular}

*The mean difference is significant at the 0.05 level. 
Table 6: Multiple Intergroup Comparison of Groups A, B and C with Respect to VAS for Air Blast Test Scores at Baseline, $3^{\text {rd }}$ and $7^{\text {th }}$ day by Tukey HSD Test

\begin{tabular}{|c|c|c|c|c|}
\hline Time Points & Groups & $\begin{array}{c}\text { Intergroup } \\
\text { Comparison }\end{array}$ & $\begin{array}{c}\text { Mean } \pm \text { Standard } \\
\text { Deviation }\end{array}$ & $\begin{array}{l}P \text { value } \\
p \leq 0.05\end{array}$ \\
\hline \multirow[t]{4}{*}{ Baseline } & \multirow[t]{2}{*}{ Group A } & Group B & $-0.19 \pm 0.36$ & 0.848 \\
\hline & & Group C & $0.05 \pm 0.36$ & 0.989 \\
\hline & \multirow[t]{2}{*}{ Group B } & Group A & $0.19 \pm 0.36$ & 0.848 \\
\hline & & Group C & $0.25 \pm 0.36$ & 0.771 \\
\hline & \multirow[t]{2}{*}{ Group C } & Group A & $-0.05 \pm 0.36$ & 0.989 \\
\hline & & Group B & $-0.25 \pm 0.36$ & 0.771 \\
\hline \multirow[t]{6}{*}{$3^{\text {rd day }}$} & \multirow[t]{2}{*}{ Group A } & Group B & $-1.23 \pm 0.33$ & $0.001 *$ \\
\hline & & Group C & $-1.55 \pm 0.33$ & $0.000 *$ \\
\hline & \multirow[t]{2}{*}{ Group B } & Group A & $1.23 \pm 0.33$ & $0.001^{*}$ \\
\hline & & Group C & $-0.32 \pm 0.33$ & 0.586 \\
\hline & \multirow[t]{2}{*}{ Group C } & Group A & $1.55 \pm 0.33$ & $0.000^{*}$ \\
\hline & & Group B & $0.32 \pm 0.33$ & 0.586 \\
\hline \multirow[t]{6}{*}{$7^{\text {th }}$ day } & \multirow[t]{2}{*}{ Group A } & Group B & $-1.23 \pm 0.24$ & $0.000 *$ \\
\hline & & Group C & $-2.54 \pm 0.24$ & $0.000 *$ \\
\hline & \multirow[t]{2}{*}{ Group B } & Group A & $1.23 \pm 0.23$ & $0.000 *$ \\
\hline & & Group C & $-1.30 \pm 0.23$ & $0.000 *$ \\
\hline & \multirow[t]{2}{*}{ Group C } & Group A & $2.54 \pm 0.23$ & $0.000 *$ \\
\hline & & Group B & $1.30 \pm 0.23$ & $0.000^{*}$ \\
\hline
\end{tabular}

*The mean difference is significant at the 0.05 level.

\section{Discussion}

Several strategies were used to treat hypersensitivity including specific dentifrices, dentin adhesives, antibacterial agents, aldehydes, resin suspensions, fluoride rinses, fluoride varnishes, calcium phosphate, potassium nitrate, oxalates and strontium agents. More recently, dentin desensitizing solutions also have been used under amalgam restorations and crowns to prevent postoperative sensitivity. Also, Nd: YAG laser, bioactive glass, Portland cement and casein phosphopeptide have been used to reduce hypersensitivity ${ }^{(10)}$. The stannous fluoride dentifrice assessed in the present study as a desensitizing agent [Group A] has been reported to have multiple oral care benefits like antiplaque (11), antigingivitis ${ }^{(12)}$, stain inhibition ${ }^{(13)}$ and improving breath malodour ${ }^{(14)}$. In this dentifrice, stannous ions are thought to occlude dentinal tubules and prevent the stimulation of nerve endings and the pain response.

In this present study, in Group A (Table 1) a statistically significant reduction of dentinal hypersensitivity was observed between baseline $(4.83 \pm 1.34)$ and $3^{\mathrm{rd}}$ day $(1.89 \pm 1.34)$ as well as between baseline and $7^{\text {th }}$ day $(0.24 \pm 0.32)$. These results were in comparison with a comparative four-week clinical study, in which stannous fluoride dentifrice was compared with sodium fluoride negative control dentifrice ${ }^{(15)}$. Also, the results were in comparison with an eight-week clinical study in which stannous fluoride showed better reduction in dentin hypersensitivity as compared to potassium nitrate positive control toothpaste $^{(16)}$. Likewise, Schiff and his collaborators carried out two studies to assess the efficacy of the stannous fluoride/sodium hexametaphosphate formula in reducing hypersensitivity. Results showed that the stannous fluoride/sodium hexametaphosphate dentifrice produced a significant decrease in sensitivity as compared to the control dentifrice ${ }^{(17)}$. These results were in comparison with our study that the stannous fluoride dentifrice showed better reduction in sensitivity.

Dentifrice based on a novel arginine-calcium carbonate technology $(8.0 \%$ arginine, calcium carbonate and $1000 \mathrm{ppm}$ fluoride as sodium monofluorophosphate) that was used in the present study [Group B] works by triggering the deposition of phosphate and physically sealing dentin tubules with a plug containing arginine, 
calcium carbonate and phosphate ${ }^{(2)}$. In 2002, Kleinberg et al have reported that reported the development of an anti-sensitivity technology ProArgin $^{\mathrm{TM}}$ based on the understanding of the role that saliva plays in naturally reducing dentin hypersensitivity over time ${ }^{(18)}$. The essential components were arginine, an amino acid positively charged at physiologic $\mathrm{pH}$ (6.5-7.5), bicarbonate, a $\mathrm{pH}$ buffer and insoluble calcium carbonate, a source of calcium. The arginine present in the product was obtained from vegetable sources.

As witnessed in Group A, statistically significant reduction of dentinal hypersensitivity was observed in Group B (Table 1) between baseline $(5.03 \pm 1.22)$ and $3^{\text {rd }}$ day $(3.12 \pm 1.17)$ as well as between baseline and $7^{\text {th }}$ day $(1.47 \pm 0.94)$. These findings were in accordance with mechanism proposed in the study by Kleinberg et al, 2002 ${ }^{(18)}$. These findings were also found in accordance with a similar study (Kakar et al, 2012) where the clinical efficacy of a Pro-Argin paste was compared with $1000 \mathrm{ppm}$ fluoride containing $2 \%$ potassium ionon reduction in dentin hypersensitivity in a randomized clinical trial ${ }^{(19)}$. The combination showed greater efficacy after two, four and eight weeks of use. In another study, Hamlin et al applied 8\% arginine along with calcium carbonate and pumice prophylaxis paste prior as control to a professional dental cleaning procedure $^{(20)}$. Sensitivity measurements were taken immediately thereafter where the results showed that occlusion led to highly significant reductions in dentin fluid flow and that the tubule plug remained resistant to normal pulpal pressure (20). Together, these results have clearly demonstrated that the arginine-calcium carbonate desensitizing paste reduces dentin hypersensitivity by sealing and plugging dentin tubules. It is simple and has been proven to show strong efficacy, fast onset and long lasting effect.

As for the Group C (control), reduction of dentinal hypersensitivity was found to be statistically significant (Table 1) between baseline (4.78 \pm $1.58)$ and $3^{\text {rd }}$ day $(3.45 \pm 1.27)$ and also between baseline and $7^{\text {th }}$ day $(2.77 \pm 1.22)$. This may be because of the mechanism proposed by Nishida in 1980 where he reported that no agents were needed in the treatment of dentin hypersensitivity. $\mathrm{He}$ also suggested that improvement of dentin hypersensitivity following periodontal surgery would occur occasionally, eventually reaching the pre surgical level. This state was known as the natural desensitization process and the natural reduction of dentin hypersensitivity may be related to the patient's oral hygiene practices. Addition to this, plaque removal through regular oral hygiene was considered an essential part of therapy for reducing hypersensitivity. The results obtained from Group C can also be attributed to the well-known placebo or Hawthorne effect. The fact that placebo products can reduce sensitivity by as much as $40 \%$ from baseline has significantly impacted the ability to differentiate the efficacy of a test product in some studies ${ }^{(21,9)}$.

Intergroup comparison among the three groups have revealed that dentin hypersensitivity reduction in Group A (stannous fluoride) was statistically significant when compared to Group $\mathrm{B}$ (arginine bicarbonate) at $3^{\text {rd }}$ and $7^{\text {th }}$ day. The results of the present study are in accordance with a previous study conducted by Tao $\mathrm{He}$ et al, $2011^{(22)}$. He compared desensitizing efficacy of stannous - containing sodium fluoride dentifrice with arginine -calcium carbonate dentifrice as a positive control. Both products provided a statistically significant reduction in sensitivity at day 3 versus baseline. However, only the stannous -containing dentifrice provided a significant benefit immediately post - brushing. The results were supported by the subject's own assessment of pain using a VAS score. In our study, the VAS score of Group A (stannous fluoride) was significantly lower than the VAS score of Group $\mathrm{B}$ (arginine bicarbonate). Within the limitations of the study, it can be suggested that stannous fluoride has exhibited a better effect in managing dentinal hypersensitivity. 


\section{Conclusion}

Dentin hypersensitivity is a relatively common problem seen in daily clinical practice. It is characterized by a sharp, transient pain in response to a sensory stimulus that affects eating, drinking and brushing teeth. This condition affects nearly millions of people worldwide with approximately one in five adults and can be seen in all age groups. Patients who have received periodontal therapy are four times more at risk of developing hypersensitivity than the general population. Epidemiologic research suggests that prevalence peaks between 30 and 40 years of age and that women experience a higher incidence of dentin hypersensitivity at a younger age than men. This is because gingival recession and exposed root surfaces occur as a result of periodontal disease and its associated therapy. Any change in fluid pressure within the tubule is detected by the odontoblast, after which the nerve becomes polarized and the patient feels pain. As individuals retain their dentition for longer and as diets change, it is reasonable to expect that there will be a higher incidence of complaints related to dentin hypersensitivity and with that an increase in requests for treatment. The treatment choices available to relieve dentin hypersensitivity are to occlude the dentin tubules or to desensitize the nerves so that they are not responsive to stimulation. Thus, there has been an increasing need for the dental team to understand the biology, etiology as well as the modalities of treatment of dentin hypersensitivity.

In this randomized double-blind clinical study including 90 patients, the results support the efficacy of stannous fluoride containing toothpaste at $3^{\text {rd }}$ and $7^{\text {th }}$ day after scaling. The effects of stannous fluoride-containing toothpaste exhibited a superior therapeutic effect compared to toothpastes containing other desensitizing components as indicated by the air blast sensitivity test. At days 3 and 7, there was a statistically significant reduction in hypersensitivity in all the three groups. On intergroup comparison, reduction in hypersensitivity was superior and statistically significant in group A when compared to the other two groups, because the stannous ions are thought to occlude dentinal tubules and prevent the stimulation of nerve endings and the pain response. In future, similar studies have to be carried out with larger sample size and longer follow-up period which may be helpful in understanding the efficiency of suitable dentifrices in combating dentin hypersensitivity.

Conflict of Interest: Declared none.

Acknowledgement: Declared none.

Sources of Support: Nil

\section{References}

1. Addy M. Dentine hypersensitivity: Definition, prevalence, distribution and etiology. In: Tooth wear and sensitivity 2000;239-248.

2. Addy M. Etiology and clinical implications of dentine hypersensitivity. Dent Clin North Amer 1990;34:503-514.

3. Gillam D.G, Orchardson R. Advances in the treatment of root dentin sensitivity: Mechanisms and treatment principles. Endod Topics 2001;13:13-33.

4. Clayton D.R, McCarthy D, Gillam D.G. A study of the prevalence and distribution of dentine sensitivity in a population of 17-58 year-old serving personnel on an RAF base in the Midlands. J Oral Rehab 2002;29:1423.

5. Canadian advisory board on dentine hypersensitivity. Consensus-based recommendations for the diagnosis and management of dentine hypersensitivity. J Can Den Assoc 2003;69:221-226.

6. Von Troil B, Needleman I, Sanz M. A systematic review of the prevalence of root sensitivity following periodontal therapy. J Clin Periodontol 2002; 29(3): 173-177.

7. Addy M. Dentin hypersensitivity: New perspectives on an old problem. Int Dent J 2002;52:367-375. 
8. Drisko C.H. Dentine hypersensitivity. Dental hygiene and periodontal considerations. Int Dent J 2002;52:385-393.

9. Orchardson R, Gillam D.G. The efficacy of potassium salts as agents for treating dentin hypersensitivity $\mathbf{J}$ Orofac Pain 2000;14:9-19.

10. Roberson T.M, Heymann H.O, Jr. Swift E.J, Sturdevant C. Fundamental concepts of enamel and dentin adhesion. Art Sci Oper Dent 2006;5:257.

11. He T, Sun L, Li S, Ji N. The anti-plaque efficacy of a novel stannous-containing sodium fluoride dentifrice: A randomized and controlled clinical trial. Am J Dent 2010;23:11B-16B.

12. Li X, Fan X, Yin W, He T, Zhang Y, Sun L. Anti-gingivitis efficacy of a stannouscontaining sodium fluoride toothpaste. J Dent Res 2011;22:1-5.

13. He T, Dunavent J.M, Fiedler S.K, Baker R.A. Arandomized clinical study to assess the extrinsic staining profiles of stannousand triclosan -containing dentifrices.Am J Dent 2010;23:22B-26B.

14. Feng X.P, Chen X, Cheng R, Sun L, Zhang Y, He T. Breath malodor reduction with use of a stannous - containing sodium fluoride dentifrice: A meta-analysis of four randomized and controlled clinical trials. Am J Dent2010;23:27B-31B.

15. Day T.N, Einwag J, Hermann J.S, He T, Anastasia M.K, Barker M, Zhang Y. A clinical assessment of the efficacy of a stannous-containing sodium fluoride dentifrice on dentinal hypersensitivity. J Contemp Dent Pract 2010;11:1-8.

16. Ni L.X, He T, Chang A, Sun L. The desensitizing efficacy of a novel stannouscontaining sodium fluoride dentifrice: An 8 week randomized and controlled clinical trial. Am J Dent2010;23:17B-21B.
17. Schiff T, Saletta L, Baker R.A, Winston J.L, He T. Desensitizing effect of a stabilized stannous fluoride/sodium hexametaphosphate dentifrice. Compend Cont Dent Educ2005;26:35-40.

18. Kleinberg I. Sensistat. A new saliva -based composition for simple and effective treatment of dentinal sensitivity pain. Dent Today 2002;21:42-47.

19. Kakar A, Kakar K, Sreenivasan P.K, DeVizio W, Kohli R. Comparison of the clinical efficacy of a new dentifrice containing $8.0 \%$ arginine, calcium carbonate and $1000 \mathrm{ppm}$ fluoride to a commercially available sensitivetoothpaste containing $2 \%$ potassium ion on dentin hypersensitivity: a randomized clinical trial. J Clin Dent 2012;23(2):40-47.

20. D. Hamlin, K. Phelan Williams, E. Delgado, Y.P. Zhang, W. DeVizio, L.R. Mateo. Introducing pro-argin ${ }^{\mathrm{TM}}-\mathrm{A}$ breakthrough technology based upon arginine and calcium for in-office treatment of dentin hypersensitivity. Am J Dent 2009;22:16A-20A.

21. Markowitz K, Pashley D.H. Discovering new treatments for sensitive teeth. The long path from biology to therapy. J Oral Rehab 2007;35:300-315.

22. Tao He, Richard Cheng, Aaron R Biesbrock. Rapid desensitizing efficacy of a stannous-containing sodium fluoride dentifrice. J Clin Dent2011;22:40-45. 